\title{
A REMARK ON CENTRAL SEQUENCE ALGEBRAS OF THE TENSOR PRODUCT OF $\mathrm{II}_{1}$ FACTORS
}

\author{
WENMING WU AND WEI YUAN \\ (Communicated by Marius Junge)
}

\begin{abstract}
Let $\mathcal{M}$ and $\mathcal{N}$ be two type $\mathrm{II}_{1}$ factors with separable predual and $\omega$ a free ultrafilter on $\mathbb{N}$. If the central sequence algebra $\mathcal{N}_{\omega}$ is abelian and there is a non-atomic abelian subalgebra $\mathcal{A}$ in $\mathcal{M}$ such that any central sequence of $\mathcal{M} \bar{\otimes} \mathcal{N}$ is contained in the ultrapower $(\mathcal{A} \bar{\otimes} \mathcal{N})^{\omega}$, then $(\mathcal{M} \bar{\otimes} \mathcal{N})_{\omega}$ is abelian. It is also shown that there is an action $\alpha$ of the free group $F_{2}$ on the group von Neumann algebra $\mathcal{L}_{\mathbb{Z}}$ such that the central sequence algebra of $\mathcal{M}=\mathcal{L}_{\mathbb{Z}} \rtimes_{\alpha} F_{2}$ is abelian and non-trivial and any central sequence in $\mathcal{M} \bar{\otimes} \mathcal{N}$ is in the ultrapower $\left(\mathcal{L}_{\mathbb{Z}} \bar{\otimes} \mathcal{N}\right)^{\omega}$.
\end{abstract}

\section{INTRODUCTION AND PRELIMINARIES}

Von Neumann algebras are strong-operator closed self-adjoint subalgebras of the algebra of all bounded linear operators acting on a Hilbert space. Factors are von Neumann algebras whose center consists of the scalar multiples of the identity operator. Every von Neumann algebra is the direct sum or direct integral of factors. Thus factors are the building blocks for all von Neumann algebras. Murray and von Neumann classified factors by means of a relative dimension function. Finite factors are those for which this dimension function has a bounded range. The infinite dimensional finite factors are referred to as the type $\mathrm{II}_{1}$ factors. By using the ideas of central sequences, Murray and von Neumann gave examples of nonisomorphic factors of type $\mathrm{II}_{1}$.

Let $\mathcal{M}$ be a $\mathrm{II}_{1}$ factor with the unique faithful normal tracial state $\tau$. The $2-$ norm on $\mathcal{M}$ is given by $\|T\|_{2}=\tau\left(T^{*} T\right)^{1 / 2}, \forall T \in \mathcal{M}$. A uniform bounded sequence $\left\{T_{n}\right\}$ in $\mathcal{M}$ is said to be a central sequence if $\lim _{n \rightarrow \infty}\left\|T_{n} A-A T_{n}\right\|_{2}=0$ for any $A$ in $\mathcal{M}$. It is easy to see that any subsequence of a central sequence is also central. A central sequence $\left\{T_{n}\right\}$ is said to be non-trivial if $\left\|T_{n}-\tau\left(T_{n}\right) I\right\|_{2} \nrightarrow 0$ as $n$ tends to infinity.

Suppose $\omega$ is a free ultrafilter of $\mathbb{N}$. Let $l^{\infty}(\mathcal{M})$ be the set of all uniform bounded sequences in $\mathcal{M}$. Then $l^{\infty}(\mathcal{M})$ is a von Neumann algebra (with pointwise multiplication) and $I_{\omega}=\left\{\left\{T_{n}\right\} \in l^{\infty}(\mathcal{M}): \lim _{n \rightarrow \omega}\left\|T_{n}\right\|_{2}=0\right\}$ is a maximal ideal in $l^{\infty}(\mathcal{M})$. The ultrapower $\mathcal{M}^{\omega}$ of $\mathcal{M}$, which is defined by $\mathcal{M}^{\omega}=l^{\infty}(\mathcal{M}) / I_{\omega}$, is a $\mathrm{II}_{1}$ factor $\left[9 . \mathcal{M}\right.$ is canonically embedded in $\mathcal{M}^{\omega}$ as constant sequences given by elements in $\mathcal{M}$. Therefore, the algebra formed by central sequences of $\mathcal{M}$ can be viewed as the relative commutant of $\mathcal{M}$ in $\mathcal{M}^{\omega}$ and denoted by $\mathcal{M}_{\omega}$.

Received by the editors November 18, 2011 and, in revised form, September 10, 2012.

2010 Mathematics Subject Classification. Primary 46L10.

This work was partially supported by NSFC (No.11271390, No. 11301511) and Natural Science Foundation Project of CQ CSTC (No. CSTC, 2010BB9318). 
Dixmier 2] proved that $\mathcal{M}_{\omega}$ is either trivial or non-atomic. In [5], McDuff showed that $\mathcal{M}_{\omega}$ is non-commutative if and only if $\mathcal{M} \cong \mathcal{M} \otimes \mathcal{R}$, where $\mathcal{R}$ is the hyperfinite $\mathrm{II}_{1}$ factor. Such a factor is thus called a McDuff factor. McDuff also proved in the same paper that the central sequence algebra of a McDuff factor is type $\mathrm{II}_{1}$.

Suppose that $\mathcal{M}$ and $\mathcal{N}$ are two $\mathrm{II}_{1}$ factors. It is clear that if $\mathcal{M}_{\omega}$ or $\mathcal{N}_{\omega}$ is noncommutative, then $(\mathcal{M} \otimes \mathcal{N})_{\omega}$ is also non-commutative. However, if both $\mathcal{M}_{\omega}$ and $\mathcal{N}_{\omega}$ are abelian, it is not known whether $(\mathcal{M} \otimes \mathcal{N})_{\omega}$ is still abelian. The full factor is the factor which does not have property $\Gamma$, i.e., the central sequence algebra of the factor is trivial. A. Connes proved that the tensor product of two $\mathrm{II}_{1}$ factors is full if and only if both the two factors are full ([1]). In [3], Fang, Ge and Li showed that $\mathcal{R}_{\omega}$ is prime, which implies that the central sequence algebra of the tensor product of two factors may not be the tensor product of the central sequence algebras of the factors respectively.

In this paper, we will try to shed more light on the question of whether $(\mathcal{M} \otimes \mathcal{N})_{\omega}$ is abelian if both $\mathcal{M}_{\omega}$ or $\mathcal{N}_{\omega}$ are abelian. More precisely, we will prove in section 2 that if $\mathcal{N}_{\omega}$ is abelian and there exists a non-atomic abelian von Neumann subalgebra $\mathcal{A}$ of $\mathcal{M}$ such that $(\mathcal{M} \bar{\otimes} \mathcal{N})_{\omega} \subset(\mathcal{A} \bar{\otimes} \mathcal{N})^{\omega}$, then $(\mathcal{M} \bar{\otimes} \mathcal{N})_{\omega}$ is abelian. In the last section, we will construct a $\mathrm{II}_{1}$ factor $\mathcal{M}$ with a non-trivial abelian central sequence algebra such that any central sequence of $\mathcal{M} \bar{\otimes} \mathcal{N}$ is contained in $(\mathcal{A} \bar{\otimes} \mathcal{N})^{\omega}$, where $\mathcal{A}$ is a non-atomic abelian von Neumann subalgebra of $\mathcal{M}$ and $\mathcal{N}$ is any other $\mathrm{II}_{1}$ factor.

\section{MAin RESUlt}

Throughout this section, we let $\omega$ be a free ultrafilter on $\mathbb{N}$ and let $\mathcal{M}_{\omega}, \mathcal{N}_{\omega}$ be the central sequence algebras of $\mathrm{II}_{1}$ factors $\mathcal{M}$ and $\mathcal{N}$. For simplicity of notation, we use the same letter $\tau$ to denote the faithful normal tracial state on $\mathcal{M}, \mathcal{N}$ and $\mathcal{M} \bar{\otimes} \mathcal{N}$.

Theorem 2.1. With notation as above, if $\mathcal{N}_{\omega}$ is abelian and there is a non-atomic abelian von Neumann subalgebra $\mathcal{A}$ of $\mathcal{M}$ such that any central sequence in $\mathcal{M} \bar{\otimes} \mathcal{N}$ is contained in the ultrapower $(\mathcal{A} \bar{\otimes} \mathcal{N})^{\omega}$, then $(\mathcal{M} \bar{\otimes} \mathcal{N})_{\omega}$ is abelian.

We will prove this fact by showing that any two central sequences in $\mathcal{M} \bar{\otimes} \mathcal{N}$ are commuting [5].

Assume that $\mathcal{M}$ and $\mathcal{N}$ act on the separable Hilbert spaces $\mathcal{H}$ and $\mathcal{K}$ respectively. Since $\mathcal{A}$ is a non-atomic abelian von Neumann subalgebra of $\mathcal{M}$, there is a separable Hilbert space $\mathcal{H}_{2}$ and a unitary operator $W$ from $\mathcal{H}$ onto $\mathcal{H}_{2} \otimes L^{2}([0,1], \mu)$ such that $W \mathcal{A} W^{*}=I \otimes L^{\infty}([0,1], \mu)$, where $\mu$ is the Lebesgue measure on the interval $[0,1]\left([4)\right.$. Moreover, if $A \in \mathcal{A}$ and $W A W^{*}=I \otimes \varphi(A)$, we have $\tau(A)=$ $\int_{[0,1]} \varphi(A)(x) d \mu(x)$. We will therefore assume in the rest of this section that $\mathcal{H} \otimes \mathcal{K}=\mathcal{H}_{2} \otimes L^{2}([0,1], \mu) \otimes \mathcal{K}$ and $\mathcal{A}=I \otimes L^{\infty}([0,1], \mu)$.

Note that $L^{2}([0,1], \mu) \otimes \mathcal{K}$ can be identified with $L^{2}([0,1], \mathcal{K})([10])$. The inner product on $L^{2}([0,1], \mathcal{K})$ is given by

$$
\langle\xi, \eta\rangle=\int_{[0,1]}\langle\xi(x), \eta(x)\rangle d \mu(x), \quad \xi, \eta \in L^{2}([0,1], \mathcal{K}) .
$$

Lemma 2.2. If a sequence $\left\{\xi_{n}\right\}$ converges to $\xi$ in $L^{2}([0,1], \mathcal{K})$, then there exists a subsequence $\left\{\xi_{n_{k}}\right\}$ of $\left\{\xi_{n}\right\}$ such that $\left\{\xi_{n_{k}}(x)\right\}$ converges to $\xi(x)$ for almost every $x$ in $[0,1]$. 
Proof. If $\left\{\xi_{n}\right\}$ converges to $\xi$, we have

$$
\int_{[0,1]}\left\|\xi(x)-\xi_{n}(x)\right\|^{2} d \mu(x) \rightarrow 0, \quad n \rightarrow \infty .
$$

It is easy to see that (2.1) implies the lemma ([11]).

Before we proceed further, let us recall the following fact about operators in $L^{\infty}([0,1]) \bar{\otimes} \mathcal{N}$. For any $T \in L^{\infty}([0,1]) \bar{\otimes} \mathcal{N}$, there exists a $\mathcal{N}$-valued bounded function $T(\cdot)$ on $[0,1]$ such that, for any $\mathcal{K}$-valued measurable function $\xi(\cdot)$, the functions $x \in[0,1] \rightarrow T(x) \xi(x) \in \mathcal{K}$ and $x \in[0,1] \rightarrow T(x)^{*} \xi(x) \in \mathcal{K}$ are measurable and $(T \xi)(x)=T(x) \xi(x)$ for almost every $x \in[0,1]$. Moreover, we have

$$
\begin{aligned}
\|T\|_{2} & =\tau\left(T^{*} T\right)^{\frac{1}{2}}=\left(\int_{[0,1]} \tau\left(T(x)^{*} T(x)\right) d \mu(x)\right)^{\frac{1}{2}} \\
& =\left(\int_{[0,1]}\|T(x)\|_{2}^{2} d \mu(x)\right)^{\frac{1}{2}} .
\end{aligned}
$$

It follows immediately that for almost every $x \in[0,1]$,

$$
\begin{aligned}
& ((I \otimes T) \xi)(x)=T \xi(x), \quad T \in \mathcal{N}, \\
& ((f \otimes I) \xi)(x)=f(x) \xi(x), \quad f \in L^{\infty}([0,1]) .
\end{aligned}
$$

We refer the reader to [10] and [4] for a more detailed discussion on the direct integral of Hilbert spaces and von Neumann algebras.

To prove Theorem 2.1, we will need the following technical lemma.

Lemma 2.3. If $\left\{I \otimes T_{n}\right\} \in\left(I \otimes L^{\infty}([0,1], \mu) \otimes \mathcal{N}\right)^{\omega}$ is a central sequence of $\mathcal{M} \bar{\otimes} \mathcal{N}$, then there is a subsequence $\left\{R_{n}\right\}$ of $\left\{T_{n}\right\}$ such that $\left\{R_{n}(x)\right\} \in \mathcal{N}_{\omega}$ for almost every $x \in[0,1]$.

Proof. The proof is an easy application of the diagonal argument. Without loss of generality, we could assume that $\left\|T_{n}\right\|=1, \forall n \in \mathbb{N}$. Since $\left\{I \otimes T_{n}\right\}$ is a central sequence, for any $A \in \mathcal{N}$ we have

$$
\left\|T_{n}(I \otimes A)-(I \otimes A) T_{n}\right\|_{2}^{2}=\int_{[0,1]}\left\|T_{n}(x) A-A T_{n}(x)\right\|_{2}^{2} d \mu(x) \rightarrow 0, n \rightarrow \infty .
$$

Since $\mathcal{N}$ has separable predual, we can pick a $\|\cdot\|_{2}-$ dense sequence $\left\{A_{k}\right\}$ in the unit ball of $\mathcal{N}$.

By (2.2) and a straightforward inductive argument, we find subsequences $\left\{\left\{T_{n}^{(k)}\right\}\right\}_{k}$ of $\left\{T_{n}\right\}$ such that $\left\{T_{n}^{(k+1)}\right\}$ is a subsequence of $\left\{T_{n}^{(k)}\right\}$, and for almost every $x \in[0,1]$ we have

$$
\left\|T_{n}^{(k)}(x) A_{i}-A_{i} T_{n}^{(k)}(x)\right\|_{2}^{2} \rightarrow 0, \quad n \rightarrow \infty, \quad i=1,2, \cdots, k .
$$

Let $R_{n}=T_{n}^{(n)}$ for $n=1,2, \ldots$ Then for almost every $x \in[0,1]$ we have

$$
\left\|R_{n}(x) A_{k}-A_{k} R_{n}(x)\right\|_{2}^{2} \rightarrow 0, \quad n \rightarrow \infty, \quad k=1,2, \ldots
$$

This implies that $\left\{R_{n}(x)\right\}$ is in $\mathcal{N}_{\omega}$ a.e.

We are now ready to prove Theorem 2.1. 
Proof of Theorem 2.1. Recall that we identified $\mathcal{A}$ with $I \otimes L^{\infty}([0,1], \mu)$, and all central sequences of $\mathcal{M} \bar{\otimes} \mathcal{N}$ are contained in $\left(I \otimes L^{\infty}([0,1], \mu) \otimes \mathcal{N}\right)^{\omega}$. Suppose now that $\left\{I \otimes T_{n}\right\}$ and $\left\{I \otimes S_{n}\right\}$ are two such central sequences. Then $\left\{a_{n}=\right.$ $\left.\left\|T_{n} S_{n}-S_{n} T_{n}\right\|_{2}\right\}_{n=1}^{\infty}$ is a bounded sequence since $0 \leq a_{n} \leq 2\left\|\left\{T_{n}\right\}\right\|\left\|\left\{S_{n}\right\}\right\|$.

We claim that for any subsequence $\left\{n_{k}\right\}_{k=1}^{\infty}$ of $\mathbb{N}$, there is a subsequence $\left\{m_{k}\right\}_{k=1}^{\infty}$ of $\left\{n_{k}\right\}_{k=1}^{\infty}$ such that $a_{m_{k}} \rightarrow 0$ as $k \rightarrow \infty$. It is clear that the claim implies the theorem.

To prove the claim, let $\left\{n_{k}\right\}_{k=1}^{\infty}$ be a subsequence of $\mathbb{N}$. Note that every subsequence of a central sequence is still a central sequence. Hence, by Lemma 2.3, there exists a subsequence $\left\{m_{k}\right\}$ of $\left\{n_{k}\right\}$ such that both $\left\{T_{m_{k}}(x)\right\}$ and $\left\{S_{m_{k}}(x)\right\}$ are in $\mathcal{N}_{\omega}$ almost everywhere.

Let $f_{k}$ be the function defined by

$$
f_{k}(x)=\left\|T_{m_{k}}(x) S_{m_{k}}(x)-S_{m_{k}}(x) T_{m_{k}}(x)\right\|_{2}, \quad x \in[0,1], \quad k=1,2, \ldots
$$

The functions $\left\{f_{k}\right\}$ are measurable since the $\mathcal{N}$-valued functions $T_{n}(x)$ and $S_{n}(x)$ are $\mu$-measurable. Moreover, $\left\{f_{k}\right\}$ are bounded by the boundedness of $\left\{T_{n}\right\}$ and $\left\{S_{n}\right\}$.

Since $\mathcal{N}_{\omega}$ is abelian and $\left\{S_{m_{k}}(x)\right\}$ and $\left\{T_{m_{k}}(x)\right\}$ are in $\mathcal{N}_{\omega}$ almost everywhere, then $f_{k}(x) \rightarrow 0(k \rightarrow \infty)$ almost everywhere. Hence, by the Lesbegue dominated converge theorem, we have

$$
\lim _{k \rightarrow \infty} a_{m_{k}}=\lim _{k \rightarrow \infty}\left(\int_{[0,1]}\left|f_{k}(x)\right|^{2} d \mu(x)\right)^{\frac{1}{2}}=\left(\int_{[0,1]} \lim _{k \rightarrow \infty}\left|f_{k}(x)\right|^{2} d \mu(x)\right)^{\frac{1}{2}}=0,
$$

and the claim holds.

Remark 2.4. Under the conditions of Theorem 2.1, if the $\mathrm{II}_{1}$ factors $\mathcal{M}$ and $\mathcal{N}$ have the property $\Gamma$ and are not McDuff factors, then their tensor product $\mathcal{M} \bar{\otimes} \mathcal{N}$ also has the property $\Gamma$ and is not a McDuff factor.

\section{The CROSSED PRODUCT $\mathcal{L}_{\mathbb{Z}} \rtimes_{\alpha} F_{2}$}

To construct the $\mathrm{II}_{1}$ factor mentioned in the introduction, we need to first fix some notation. Let $F_{2}$ be the free group with two generators $a$ and $b$. The group factor $\mathcal{L}_{F_{2}}$ is constructed by completing the left regular representation of $F_{2}$ on the Hilbert space $l^{2}\left(F_{2}\right)$ in the weak operator topology. We use $L_{g}, g \in F_{2}$, to denote the unitary operator corresponding to the group element $g$ in $\mathcal{L}_{F_{2}}$. It is clear that $L_{a}$ and $L_{b}$ generate $\mathcal{L}_{F_{2}}$.

Let $\left\{e_{n}\right\}_{n \in \mathbb{Z}}$ be the canonical orthonormal basis of $l^{2}(\mathbb{Z})$, i.e., $e_{n}(m)=\delta_{n, m}$. We use $E_{n}$ to denote the orthogonal projection from $l^{2}(\mathbb{Z})$ onto the one dimensional subspace spanned by $e_{n}$. The bilateral shift operator $U$ on $l^{2}(\mathbb{Z})$ is given by $U e_{n}=$ $e_{n+1}$. It is well known that $U$ is a Haar unitary with respect to the vector state $\omega_{e_{0}}$ associated with $e_{0}$ (which is defined on all of $B\left(l^{2}(\mathbb{Z})\right)$ ), i.e., $\omega_{e_{0}}(A)=\left\langle A e_{0}, e_{0}\right\rangle$. Let $\mathcal{L}_{\mathbb{Z}}$ be the von Neumann algebra generated by the operator $U$.

Since every unitary pair determines a representation of $F_{2}$, we now construct a unitary representation $\pi$ of $F_{2}$ by giving such a pair of unitaries on $l^{2}(\mathbb{Z})$ :

$$
V_{a} e_{n}=e_{n}, \quad V_{b} e_{n}=e^{-i n \theta} e_{n}, \quad n \in \mathbb{Z},
$$

where $\frac{\theta}{2 \pi} \in[0,1) \backslash \mathbb{Q}$. For the rest of this section, we will use $V_{g}$ to denote $\pi(g)$. It is easy to see that $V_{b} E_{n}=e^{-i n \theta} E_{n}$ and $V_{b} U V_{b}^{-1}=e^{-i \theta} U$. Thus $\pi$ induces an automorphic representation $\alpha$ of $F_{2}$ on $\mathcal{L}_{\mathbb{Z}}: \alpha_{g}(A)=V_{g} A V_{g}^{*}, g \in F_{2}, A \in \mathcal{L}_{\mathbb{Z}}$. 
Remember that every element in $\mathcal{L}_{\mathbb{Z}} \rtimes_{\alpha} F_{2}$ can be written as $\sum_{g \in F_{2}} x_{g} V_{g} \otimes L_{g}$, where $x_{g} \in\{U\}^{\prime \prime}$ and $\sum_{g \in F_{2}} \omega_{e_{0}}\left(x_{g}^{*} x_{g}\right)<\infty$. Let $\tau_{0}\left(\sum_{g \in F_{2}} x_{g} V_{g} \otimes L_{g}\right) \triangleq \omega_{e_{0}}\left(x_{e}\right)$. It is routine to check that $\tau_{0}$ is a faithful normal tracial state of $\mathcal{L}_{\mathbb{Z}} \rtimes_{\alpha} F_{2}$. Thus $\mathcal{L}_{\mathbb{Z}} \rtimes_{\alpha} F_{2}$ is finite. Note that the action is ergodic but not free. So the first thing we need to show is that $\mathcal{L}_{\mathbb{Z}} \rtimes_{\alpha} F_{2}\left(=\left\{U \otimes I, V_{g} \otimes L_{g}\right\}^{\prime \prime}\right)$ is a factor of type $\mathrm{II}_{1}$. More specifically, we have $\mathcal{L}_{\mathbb{Z}} \rtimes_{\alpha} F_{2}$ is a semi-solid $\mathrm{II}_{1}$ factor. Recall that a (finite) von Neumann algebra $\mathcal{M}$ is called solid (semi-solid) [6] if the relative commutant $\mathcal{A}^{\prime} \cap \mathcal{M}$ of any diffuse von Neumann subalgebra (type $\mathrm{II}_{1}$ von Neumann subalgebra) $\mathcal{A}$ is injective.

Proposition 3.1. With the above notation, $\mathcal{L}_{\mathbb{Z}} \rtimes_{\alpha} F_{2}$ is a type $I I_{1}$ factor and is semi-solid but not solid. In particular, $\mathcal{L}_{\mathbb{Z}} \rtimes_{\alpha} F_{2}$ is prime, i.e., it is not isomorphic to the tensor product of any two factors of type $I I_{1}$.

Proof. To prove $\mathcal{L}_{\mathbb{Z}} \rtimes_{\alpha} F_{2}$ is a factor, let $\sum_{g \in F_{2}} x_{g} V_{g} \otimes L_{g}$ be an element in the center. Then

$$
\sum_{g \in F_{2}} x_{g} V_{g} \otimes L_{g}=\left(V_{a} \otimes L_{a}\right)\left(\sum_{g \in F_{2}} x_{g} V_{g} \otimes L_{g}\right)\left(V_{a} \otimes L_{a}\right)^{*}=\sum_{g \in F_{2}} x_{a^{-1} g a} V_{g} \otimes L_{g} .
$$

This implies that for any $g \in F_{2}$, we have $x_{g}=x_{a^{-1} g a}$. If $b^{ \pm 1}$ appears in the reduced form of $h$, then $x_{h}$ must be 0 . Otherwise, $\sum_{g \in F_{2}} \omega_{e_{0}}\left(x_{g}^{*} x_{g}\right)=\infty$. Therefore, we now assume that the central element has the form $\sum_{n \in \mathbb{Z}} x_{a^{n}} V_{a^{n}} \otimes L_{a^{n}}$. Similarly, by considering the equations

$$
\begin{aligned}
\sum_{n \in \mathbb{Z}} x_{a^{n}} V_{a^{n}} \otimes L_{a^{n}} & =\left(V_{b} \otimes L_{b}\right)\left(\sum_{n \in \mathbb{Z}} x_{a^{n}} V_{a^{n}} \otimes L_{a^{n}}\right)\left(V_{b} \otimes L_{b}\right)^{*} \\
& =\sum_{n \in \mathbb{Z}} \alpha_{b}\left(x_{a^{n}}\right) V_{b a^{n} b^{-1}} \otimes L_{b a^{n} b^{-1}},
\end{aligned}
$$

we have $x_{g}=0$ for all $g \in F_{2} \backslash\{e\}$, where $e$ is the group unit of $F_{2}$. Hence $\mathcal{L}_{\mathbb{Z}} \rtimes F_{2}$ is a factor since $\left(V_{b} \otimes L_{b}\right)^{\prime} \cap\left(\mathcal{L}_{\mathbb{Z}} \otimes I\right)$ is trivial.

$\mathcal{L}_{\mathbb{Z}} \rtimes_{\alpha} F_{2}$ is semi-solid because $F_{2}$ is a word-hyperbolic group and the action $\alpha$ on $\mathcal{L}_{\mathbb{Z}}$ is trace-preserving (Theorem 4.6. in [7]). However, it is clear that $V_{a} \otimes L_{a}$ and $V_{b a b^{-1}} \otimes L_{b a b^{-1}}\left(=I \otimes L_{b a b^{-1}}\right)$ are in $\left(\mathcal{L}_{\mathbb{Z}} \otimes I\right)^{\prime} \cap\left(\mathcal{L}_{\mathbb{Z}} \rtimes_{\alpha} F_{2}\right)$ and that the von Neumann algebra generated by these two operators is isomorphic to the free group factor $\mathcal{L}_{F_{2}}$, thus not injective. Therefore, $\mathcal{L}_{\mathbb{Z}} \rtimes_{\alpha} F_{2}$ is not solid. Because any semi-solid $\mathrm{II}_{1}$ factor is prime, $\mathcal{L}_{\mathbb{Z}} \rtimes_{\alpha} F_{2}$ is prime.

The proof of the last proposition implies that the subalgebra $\mathcal{A}$ generated by $U \otimes I$ and $V_{a} \otimes L_{a}$ is a maximal abelian von Neumann subalgebra of $\mathcal{L}_{\mathbb{Z}} \rtimes_{\alpha} F_{2}$. Next we will show that $\mathcal{A}$ is actually a singular M.A.S.A, i.e., $\mathcal{N}(\mathcal{A})(:=\{W: W$ is a unitary in $\mathcal{M}$ such that $\left.W \mathcal{A} W^{*}=\mathcal{A}\right\}$ ) is in $\mathcal{A}$.

Proposition 3.2. $\mathcal{A}=\left\{U \otimes I, V_{a} \otimes L_{a}\right\}^{\prime \prime}$ is a singular maximal abelian von Neumann subalgebra of $\mathcal{L}_{\mathbb{Z}} \rtimes_{\alpha} F_{2}$.

Proof. Let $\mathcal{A}_{0}$ be the von Neumann subalgebra of $\mathcal{A}$ generated by the operator $V_{a} \otimes L_{a} . \quad \mathcal{A}_{0}$ is non-atomic since $V_{a} \otimes L_{a}$ is a Haar unitary. For any element $g \in F_{2}$ whose reduced form contains the letter $b$, it is not hard to check that $\left(U^{n} V_{g} U^{m} \otimes L_{g}\right) \mathcal{A}_{0}\left(U^{n} V_{g} U^{m} \otimes L_{g}\right)^{*} \perp \mathcal{A}$, i.e., $\tau_{0}\left(N_{1} N_{2}\right)=\tau_{0}\left(N_{1}\right) \tau_{0}\left(N_{2}\right)$ for any $N_{1} \in\left(U^{n} V_{g} U^{m} \otimes L_{g}\right) \mathcal{A}_{0}\left(U^{n} V_{g} U^{m} \otimes L_{g}\right)^{*}$ and $N_{2} \in \mathcal{A}$, where $\tau$ is the faithful 
normal trace defined in the discussion before Proposition 3.1 and $n, m \in \mathbb{Z}$. Thus $\tau_{0}\left(\left(U^{n} V_{g} U^{m} \otimes L_{g}\right) T\right)=\tau_{0}\left(U^{n} V_{g} U^{m} \otimes L_{g}\right) \tau_{0}(T)=0$ for any $T \in \mathcal{N}(\mathcal{A})^{\prime \prime}([\underline{8}])$.

We claim that $\mathcal{N}(\mathcal{A})^{\prime \prime} \subseteq \mathcal{A}$. Indeed, if $T=\sum_{g \in F_{2}} t_{g} V_{g} \otimes L_{g} \in \mathcal{N}(\mathcal{A})^{\prime \prime} \backslash \mathcal{A}$, then there is an element $g_{0} \in F_{2}$ with the letter $b$ in its reduced form and $t_{g_{0}} \neq 0$. As $t_{g_{0}}$ is in $\mathcal{L}_{\mathbb{Z}}$, there is an integer $k$ such that $\omega_{e_{0}}\left(U^{k} t_{g_{0}}\right) \neq 0$. Thus we have a contradiction:

$$
0=\tau_{0}(T) \tau_{0}\left(V_{g_{0}^{-1}} U^{k} \otimes L_{g_{0}^{-1}}\right)=\tau_{0}\left(T\left(V_{g_{0}^{-1}} U^{k} \otimes L_{g_{0}^{-1}}\right)\right)=\omega_{e_{0}}\left(t_{g_{0}} U^{k}\right) \neq 0,
$$

and the proposition is proved.

Proposition 3.3. The central sequence algebra $\left(\mathcal{L}_{\mathbb{Z}} \rtimes_{\alpha} F_{2}\right)_{\omega}$ is abelian and nontrivial, where $\omega$ is a free ultrafilter on $\mathbb{N}$.

Proof. Suppose that $\left\{t_{n}=\sum_{g \in F_{2}} x_{g}^{(n)} V_{g} \otimes L_{g}\right\} \in\left(\mathcal{L}_{\mathbb{Z}} \rtimes_{\alpha} F_{2}\right)^{\omega}$ is a non-trivial central sequence, where $x_{g}^{(n)} \in \mathcal{L}_{\mathbb{Z}}$. Without loss of generality, we can assume that $\left\|t_{n}\right\|=1$ and $\tau\left(t_{n}\right)=0$.

Let $S=\left\{g \in F_{2}\right.$ : the reduced form of $g$ beginning with $\left.b^{ \pm 1}\right\}$. If $\left\|\left.t_{n}\right|_{S}\right\|_{2} \nrightarrow 0$, then there is a subsequence $\left\{t_{n_{k}}\right\}$ of $\left\{t_{n}\right\}$ such that

$$
\lim _{k \rightarrow \infty} \sum_{g \in S}\left\|x_{g}^{\left(n_{k}\right)}\right\|_{2}^{2}=c>0 .
$$

Since any subsequence of a central sequence is also a central sequence, we might as well assume that $\sum_{g \in S}\left\|x_{g}^{(n)}\right\|_{2}^{2} \rightarrow c$. Let $n_{0}$ be an integer such that $\left\|\left.t_{n}\right|_{S}\right\|_{2}^{2}=$ $\sum_{g \in S}\left\|x_{g}^{(n)}\right\|_{2}^{2}>\frac{c}{2}$ whenever $n>n_{0}$.

Let $\delta=\frac{c}{8}$ and $N$ be a positive integer satisfying $\frac{c N}{4}>1$. Since $\left\{t_{n}\right\}$ is a central sequence, there is an integer $n_{1} \geq n_{0}$ such that

$$
\left\|\left(V_{a} \otimes L_{a}\right)^{i} t_{n}\left(V_{a} \otimes L_{a}\right)^{-i}-t_{n}\right\|_{2}<\delta, \quad i=1,2, \ldots, N, \quad n>n_{1} .
$$

Because $\left\|\left.\left(\left(V_{a} \otimes L_{a}\right)^{i} t_{n}\left(V_{a} \otimes L_{a}\right)^{-i}\right)\right|_{S}\right\|_{2}^{2}=\sum_{g \in a^{-i} S a^{i}}\left\|x_{g}^{(n)}\right\|_{2}^{2}=\left\|\left.t_{n}\right|_{a^{-i} S a^{i}}\right\|_{2}^{2}$, we have

$$
\left.||\left|t_{n}\right|_{a^{-i} S a^{i}}\left\|_{2}^{2}-\right\| t_{n}\right|_{S}\left\|_ { 2 } ^ { 2 } \left|\leqslant 2\left\|\left.\left(\left(V_{a} \otimes L_{a}\right)^{i} t_{n}\left(V_{a} \otimes L_{a}\right)^{-i}\right)\right|_{S}-\left.t_{n}\right|_{S}\right\|_{2}<2 \delta .\right.\right.
$$

This implies $\left\|\left.t_{n}\right|_{a^{-i} S a^{i}}\right\|_{2}^{2}>\left\|\left.t_{n}\right|_{S}\right\|_{2}^{2}-2 \delta, i=1,2, \ldots, N$.

Note that the sets $a^{-i} S a^{i}(i=1,2, \ldots, N)$ are pairwise disjoint. Thus we have

$$
1=\left\|t_{n}\right\| \geqslant\left\|t_{n}\right\|_{2}^{2} \geqslant \sum_{i=1}^{N}\left\|\left.t_{n}\right|_{a^{-i} S a^{i}}\right\|_{2}^{2} \geqslant N\left(\left\|\left.t_{n}\right|_{S}\right\|_{2}^{2}-2 \delta\right) \geqslant \frac{c N}{4}>1 .
$$

It is obviously a contradiction. Hence $\left\|\left.t_{n}\right|_{S}\right\|_{2} \rightarrow 0$. By substituting $\left(t_{n}-\left(\left.t_{n}\right|_{S}\right)\right)$ for $\left(t_{n}\right)$, we could assume that all $t_{n}$ vanish at the set $S$.

Now let $S_{0}=\left\{g \in F_{2}\right.$ : the reduced form of $g$ beginning with $\left.a^{ \pm 1}\right\}$. Then for any $0 \neq i \in \mathbb{Z}$, we have $\left.t_{n}\right|_{b^{i} S_{0} b^{-i}}=0$ and

$\left\|\left(V_{b} \otimes L_{b}\right)^{i} t_{n}\left(V_{b} \otimes L_{b}\right)^{-i}-t_{n}\right\|_{2}^{2} \geq\left\|\left.\left[\left(V_{b} \otimes L_{b}\right)^{i} t_{n}\left(V_{b} \otimes L_{b}\right)^{-i}-t_{n}\right]\right|_{S_{0}}\right\|_{2}^{2}=\left\|\left.t_{n}\right|_{S_{0}}\right\|_{2}^{2}$, since $\left\{t_{n}\right\}$ vanishes on $S$. Because $\left\{t_{n}\right\}$ is a central sequence, we have $\left\|\left.t_{n}\right|_{S_{0}}\right\|_{2} \rightarrow 0$ as $n \rightarrow \infty$.

Note that $F_{2}$ is the disjoint union of $S, S_{0}$ and $\{e\}$, and the above argument implies that $\left\{t_{n}=\sum_{g \in F_{2}} x_{g}^{(n)} V_{g} \otimes L_{g}\right\}$ is equivalent to $\left\{x_{e}^{(n)} V_{e} \otimes L_{e}\right\}$ in $\left(\mathcal{L}_{\mathbb{Z}} \rtimes_{\alpha}\right.$ $\left.F_{2}\right)^{\omega}$. It follows that all the centralizing sequences are contained in $\left(\mathcal{L}_{\mathbb{Z}}\right)^{\omega}$ and that 
$\left(\mathcal{L}_{\mathbb{Z}} \rtimes_{\alpha} F_{2}\right)_{\omega}$ is abelian. To finish the proof, we will construct a non-trival central sequence of $\mathcal{L}_{\mathbb{Z}} \rtimes_{\alpha} F_{2}$.

Since $\frac{\theta}{2 \pi} \in[0,1)$ is an irrational number, there is a sequence $\left\{n_{k}\right\}$ of natural numbers such that $\frac{n_{k} \theta}{2 \pi}-\left[\frac{n_{k} \theta}{2 \pi}\right] \rightarrow 0$ as $k \rightarrow \infty$, where $[x]$ is the integer part of $x$. Let $\left\{n_{k}\right\}$ be such a sequence and $t_{k}=U^{n_{k}} \otimes I$. Then $\left\|t_{k}\right\|=1, \tau\left(t_{k}\right)=0$ and $\left\|t_{k}\right\|_{2}=1$. It is obvious that $\left\{t_{k}\right\}$ commutes with $U \otimes I$ and $V_{a} \otimes L_{a}$. For $V_{b} \otimes L_{b}$, we have

$$
\left\|\left(V_{b} \otimes L_{b}\right) t_{k}\left(V_{b} \otimes L_{b}\right)^{-1}-t_{k}\right\|_{2}=\left\|\left(e^{-i n_{k} \theta}-1\right) U^{n_{k}}\right\|_{2} \rightarrow 0, \quad k \rightarrow \infty .
$$

Therefore, $\left\{t_{k}\right\}$ is a non-trivial central sequence of $\mathcal{L}_{\mathbb{Z}} \rtimes_{\alpha} F_{2}$.

Remark 3.4. The first part of the above argument is still valid for $\frac{\theta}{2 \pi}=\frac{q}{p}$, where $p$ and $q$ are mutually-prime integers. It is, however, easy to see that $U^{p} \otimes I$ is in the center of $\mathcal{L}_{\mathbb{Z}} \rtimes_{\alpha} F_{2}$; thus $\mathcal{L}_{\mathbb{Z}} \rtimes_{\alpha} F_{2}$ is not a factor anymore.

The following theorem can be proved by the same argument as in the proof of Proposition 3.3, so we omit its proof.

Theorem 3.5. Suppose that $\mathcal{N}$ is a factor of type $\mathrm{II}_{1}$ and $\omega$ is a free filter for $\mathbb{N}$. Let $\mathcal{M}=\mathcal{L}_{\mathbb{Z}} \rtimes_{\alpha} F_{2}$ and $\mathcal{A}=\mathcal{L}_{\mathbb{Z}} \otimes I$. Then any central sequence of $\mathcal{M} \bar{\otimes} \mathcal{N}$ is contained in $(\mathcal{A} \bar{\otimes} \mathcal{N})^{\omega}$.

\section{REFERENCES}

[1] A. Connes, Classification of injective factors. Cases $I I_{1}, I I_{\infty}, I I I_{\lambda}, \lambda \neq 1$, Ann. of Math. (2) 104 (1976), no. 1, 73-115. MR0454659 (56 \#12908)

[2] J. Dixmier, Quelques propriétés des suites centrales dans les facteurs de type $\mathrm{II}_{1}$ (French), Invent. Math. 7 (1969), 215-225. MR0248534 (40 \#1786)

[3] Junsheng Fang, Liming Ge, and Weihua Li, Central sequence algebras of von Neumann algebras, Taiwanese J. Math. 10 (2006), no. 1, 187-200. MR2186173 (2006k:46094)

[4] Richard V. Kadison and John R. Ringrose, Fundamentals of the theory of operator algebras. Vol. II. Advanced theory, Pure and Applied Mathematics, vol. 100, Academic Press Inc., Orlando, FL, 1986. MR859186 (88d:46106)

[5] Dusa McDuff, Central sequences and the hyperfinite factor, Proc. London Math. Soc. (3) 21 (1970), 443-461. MR0281018 (43 \#6737)

[6] Narutaka Ozawa, Solid von Neumann algebras, Acta Math. 192 (2004), no. 1, 111-117, DOI 10.1007/BF02441087. MR2079600(2005e:46115)

[7] Narutaka Ozawa, A Kurosh-type theorem for type $\mathrm{II}_{1}$ factors, Int. Math. Res. Not. 2006, Art. ID 97560, 18 pp., DOI 10.1155/IMRN/2006/97560. MR2211141 (2006m:46078)

[8] Sorin Popa, Orthogonal pairs of *-subalgebras in finite von Neumann algebras, J. Operator Theory 9 (1983), no. 2, 253-268. MR703810 (84h:46077)

[9] S. Sakai, The theory of $W^{*}$-algebras, Lecture notes, Yale University, 1962.

[10] M. Takesaki, Theory of operator algebras. I, reprint of the first (1979) edition. Encyclopaedia of Mathematical Sciences, vol. 124, Operator Algebras and Non-commutative Geometry, 5, Springer-Verlag, Berlin, 2002. MR 1873025 (2002m:46083)

[11] Elias M. Stein and Rami Shakarchi, Real analysis, Measure theory, integration, and Hilbert spaces, Princeton Lectures in Analysis, III, Princeton University Press, Princeton, NJ, 2005. MR2129625(2005k:28024)

College of Mathematical Sciences, Chongqing Normal University, Chongqing, 400047, People's Republic of China

E-mail address: wuwm@amss.ac.cn

Academy of Mathematics and Systems Science, Chinese Academy of Science, Beijing, 100084, People's Republic of China

E-mail address: wyuan@math.ac.cn 